\title{
Screening for specific biomarkers in the serum of postmenopausal osteoporosis patients using proteomic fingerprint techniques
}

\author{
WEIXING LI ${ }^{1}$, CHIBO LIU ${ }^{2}$ and HAIBAO WANG ${ }^{3}$ \\ ${ }^{1}$ Center of Laboratory Medicine, Zhejiang Provincial People's Hospital, Hangzhou, Zhejiang 310014; \\ Departments of ${ }^{2}$ Clinical Laboratory and ${ }^{3}$ Orthopedics, Taizhou Municipal Hospital, \\ Taizhou, Zhejiang 318000, P.R. China
}

Received August 9, 2012; Accepted September 20, 2012

DOI: $10.3892 /$ br.2012.12

\begin{abstract}
The aim of this study was to detect serum protein biomarkers and establish a diagnostic model for postmenopausal osteoporosis (PMOP) adopting matrix-assisted laser desorption/ionization time-of-flight mass spectrometry (MALDI-TOF MS) combined with weak cationic exchange (WCX) magnetic beads, and to study the clinical significance of the model in the early diagnosis of PMOP. Serum samples from 45 patients with PMOP, 30 patients with osteopenia and 40 healthy controls were prepared using WCX magnetic beads, and were then analyzed using a PBSII-C mass spectrometer reader. The protein spectra of the serum samples were normalized using the Ciphergen Proteinchip software. The peak labeling was performed using the Biomarker Wizard software. The specific protein biomarkers were screened using the Biomarker Pattern software to construct a diagnostic model for PMOP. A total of 138 discriminative mass-to-charge $(\mathrm{m} / \mathrm{z})$ ratios were found to be associated with PMOP. Of these, the $\mathrm{m} / \mathrm{z}$ peaks at $3167.4,4071.1,7771.7$ and 8140.5 were used to construct a diagnostic model in a training set. In a test set, the sensitivity and specificity of the model were 91.11 and $92.86 \%$, respectively. Potential protein biomarkers for PMOP were detected in patient serum using MALDI-TOF MS combined with WCX magnetic beads. This model of multiple biomarkers provided a powerful and reliable diagnostic method for PMOP diagnosis with high sensitivity and specificity.
\end{abstract}

\section{Introduction}

Postmenopausal osteoporosis (PMOP) is a bone disease resulting from low estrogen levels, causing reduced bone mass and bone microstructure degeneration and increased fragility

Correspondence to: Dr Haibao Wang, Department of Orthopedics, Taizhou Municipal Hospital, 381 Zhongshan Dong Road, Taizhou, Zhejiang 318000, P.R. China

E-mail: wanghb1962@126.com

Key words: postmenopausal osteoporosis, matrix-assisted laser desorption/ionization time-of-flight mass spectrometry, weak cationic exchange magnetic beads, protein of bones and fractures (1). It is a serious, frequently-occurring disease in older women. As estimated by the epidemiological survey in 2009, the incidence of the disease was 19.9 and $24 \%$ in women aged $>40$ and $>60$ years, respectively. Therefore, PMOP, which has become a serious public health concern, receives much attention (2).

With the advances in proteomics research, proteomics techniques show a notably high sensitivity and specificity in screening for biomarkers of various diseases. Currently, an increasing number of studies employs such techniques to investigate the disease-associated whole and differential proteins, in order to explore the composition and dynamic changes of the disease proteins at the whole level to gain a better understanding of the various physiological and pathological processes in individuals, as well as to search for highly effective diagnostic markers of various diseases. Matrix-assisted laser desorption/ionization time-of-light mass spectrometry (MALDI-TOF MS) is a recently emerged proteomics method, with the potential to detect various clinical samples, such as serum, urine, pleural effusion, ascites and number of secretions $(3,4)$. Therefore, MS technique is a milestone in clinical detection. The present study employed MALDI-TOF MS combined with weak cationic exchange (WCX) magnetic beads to analyze the normal bone mass in postmenopausal women and the serum protein fingerprint of different degrees of osteoporosis, and obtain the osteoporosis serum-associated differential protein peak to provide serum protein fingerprint evidence for early diagnosis and to explore the pathogenesis of clinical osteoporosis.

\section{Materials and methods}

Patients. The osteoporosis cases were postmenopausal women aged 50-68 years admitted to the Department of Orthopedics, in the Taizhou Municipal Hospital (Taizhou, China). Bone mineral density measurement of lumbar vertebrae L1-L4 showed osteopenia in 40 cases and PMOP in 70 cases [including 40 cases with osteoporosis (OS) and 30 cases with serious osteoporosis (OP)]. The patients who met the inclusion criteria were included in this study. The inclusion criteria were based on the bone density measured by dual-energy X-ray absorptiometry, established by the World Health Organization (WHO) in 1994: i) patients 
with reduced bone mass: $-1.0 \leq \mathrm{T}$-score $<-2.5$; ii) patients with osteoporosis: $\mathrm{T}$-score $\geq-2.5$; iii) patients with severe osteoporosis: T-score $\geq-2.5$, complicated by one or multiple fractures. T-score was calculated using the following formula: $\mathrm{T}$-score $=($ Bone mineral density for the subjects - mean bone mineral density for patients of the same gender and ethnicity)/standard deviations of the bone mineral density in patients of the same gender and ethnicity. The minimum T-score of the bone mineral density in the lumbar vertebrae L1-L4 was selected as the judgment standard. Patients who met the following criteria were excluded from this study: i) treatment history of anti-osteoporosis drugs, such as calcitonin and bisphosphonate; ii) secondary osteoporosis induced by endocrine disease, primary or metastatic bone tumor, blood or kidney disease and orthopaedic break, which affected bone metabolism and iii) drug administration, which affected bone metabolism within 3 months, such as estrogen and steroid hormones. The 70 cases of age-matched postmenopausal females aged 43-66 years with the normal range of bone mass served as the controls. The aforementioned cases were divided into the experimental and validation groups. This study was approved by the local Ethics Committee of the Taizhou Municipal Hospital. The patients and volunteers provided written informed consent for their participation. The patients and serum samples were then grouped into: the 'training' and the blinded 'test' groups (Table I). The blood samples were collected in 5-ml BD Vacutainers ${ }^{\circledR}$ without anticoagulation and allowed to clot at room temperature for up to $1 \mathrm{~h}$. The samples were then centrifuged at $4^{\circ} \mathrm{C}$ for $5 \mathrm{~min}$ at $10,000 \mathrm{rpm}$. The sera were frozen and stored at $-80^{\circ} \mathrm{C}$ for future analysis.

WCX magnetic beads analysis. Frozen serum samples were defrosted on ice and centrifuged at 20,000 rpm for $10 \mathrm{~min}$ at $4^{\circ} \mathrm{C}$. Serum samples were pretreated with WCX magnetic beads. Each serum sample $(10 \mu \mathrm{l})$ was denatured by the addition of $20 \mu 1$ of U9 buffer ( $9 \mathrm{~mol} / \mathrm{l}$ urea, $2 \%$ CHAPS) and mixed at $4^{\circ} \mathrm{C}$ for $30 \mathrm{~min}$. Each sample was then diluted in $100 \mu \mathrm{l}$ of low stringency buffer ( $0.1 \mathrm{M}$ sodium acetate, $\mathrm{pH} 4.0)$. WCX magnetic beads were activated. Then, $50 \mu \mathrm{l}$ of WCX magnetic beads $(50 \mathrm{mg} / \mathrm{ml})$ were transferred to a polymerase chain reaction (PCR) tube that was placed in a $2 \times 8$ well magnetic bead separator for $1 \mathrm{~min}$ for magnetic fixation of the WCX particles. The supernatant was aspirated and the tubes were removed from the separator. Low stringency buffer $(100 \mu \mathrm{l})$ was mixed with the magnetic beads. The magnetic beads were fixed for $1 \mathrm{~min}$ in the separator and the supernatant was aspirated. This washing procedure was repeated twice. One hundred microliters of each diluted serum sample were added on the surface of the activated magnetic beads for $60 \mathrm{~min}$ at room temperature, and then washed twice with $100 \mu 1$ of low stringency buffer. After the final washing step, bound molecules were eluted by incubation with $10 \mu \mathrm{l}$ of $0.5 \%(\mathrm{v} / \mathrm{v})$ trifluoroacetic acid (TFA). Elute (5 $\mu \mathrm{l})$ was diluted with SPA (5 $\mu \mathrm{l}$; saturated solution of sinapinic acid in $50 \%$ acetonitrile with $0.5 \%$ TFA. Two microliters of the resulting mixture were aspirated and spotted onto gold-coated ProteinChip ${ }^{\circledR}$ arrays. After air-drying for $\sim 5 \mathrm{~min}$ at room temperature, protein crystals on the chip were scanned with the ProteinChip (Model PBS IIc) reader (Ciphergen Biosystems, Fremont, CA, USA) to determine the masses and intensities of the
Table I. Clinical characteristics of the study subjects.

\begin{tabular}{lccc}
\hline Groups & Training set, $\mathrm{n}$ & Blind set, $\mathrm{n}$ & Total \\
\hline PMOP & & & \\
OS & 30 & 10 & 40 \\
OP & 15 & 15 & 30 \\
Control & & & \\
Osteopenia & 30 & 10 & 40 \\
Healthy subjects & 40 & 30 & 70 \\
Total & 115 & 65 & 180
\end{tabular}

PMOP, postmenopausal osteoporosis; OS, osteoporosis; OP, serious osteoporosis.

peaks over the range mass-to-charge $(\mathrm{m} / \mathrm{z}) 1,000-50,000$. The reader was set up as follows: mass range $(1,000-50,000 \mathrm{Da})$, optimized mass range (1,000-20,000 Da), laser intensity (190) and sensitivity (8). Mass calibration was performed using an all-in-one peptide reference standard containing vasopressin (1084.2 Da), somatostatin (1637.9 Da), bovine insulin $\beta$ chain (3495.9 Da), human insulin recombinant (5807.6 Da) and hirudin (7033.6 Da) (Ciphergen Biosystems). The default background subtraction was applied, and the peak intensities were normalized using the total ion current from a mass charge of 1,000 to 50,000 Da. A biomarker detection software package (Ciphergen Biomarker Wizards; Ciphergen Biosystems) was used to autodetect protein peaks. Protein peaks were selected based on the first pass of a signal-noise ratio of 3 and a minimum peak threshold of $20 \%$ of the spectra. This process was completed with a second pass of peak selection at $0.2 \%$ of the mass window, then the estimated peaks were added. These selected protein peaks were averaged as clusters and were exported to a commercially available software package (Biomarker Patterns; Ciphergen Biosystems) for further classification analysis.

Detection and statistical analysis. The profiling spectra of the serum samples from the training set were normalized by total ion current normalization using the Ciphergen ProteinChip Software (version 3.1). Peak labeling was performed using the Biomarker Wizard software 3.1 (Ciphergen Biosystems). A two-sample t-test was used to compare mean normalized intensities between the case and control groups. $\mathrm{P}<0.01$ was considered to indicate a statistically significant difference. The intensities of the selected peaks were then transferred to the Biomarker Pattern Software (BPS) to construct the classification model of PMOP.

The protein peak intensities of the samples in the test set were evaluated by BPS using the classification model. The osteoporosis group, and the control samples were then discriminated based on their proteomic profile characteristics. Sensitivity was defined as the probability of predicting osteoporosis group cases, and specificity was defined as the probability of predicting control samples. A positive predictive value that yielded a positive result reflected the probability of osteoporosis. 


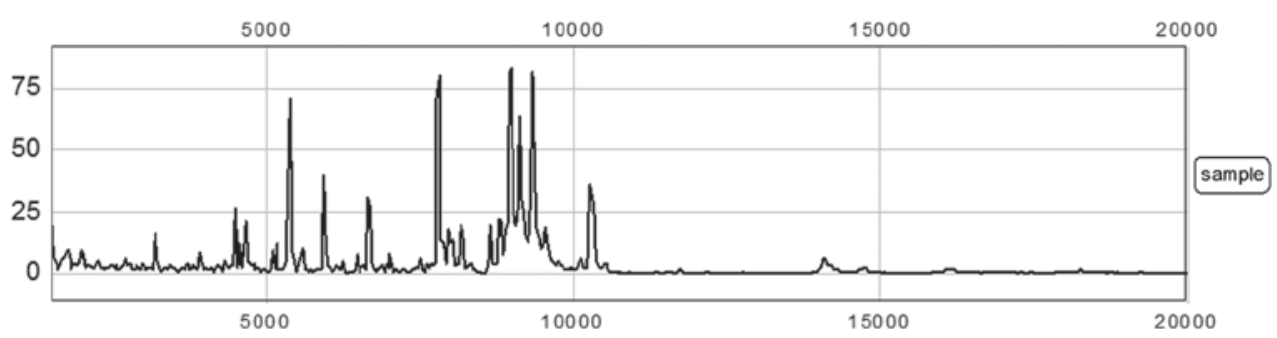

Figure 1. Representative protein spectrum of an osteoporosis sample detected by MALDI-TOF MS combined with WCX magnetic beads, showing the protein $\mathrm{m} / \mathrm{z}$ ratio between 1,000 and 20,000 .
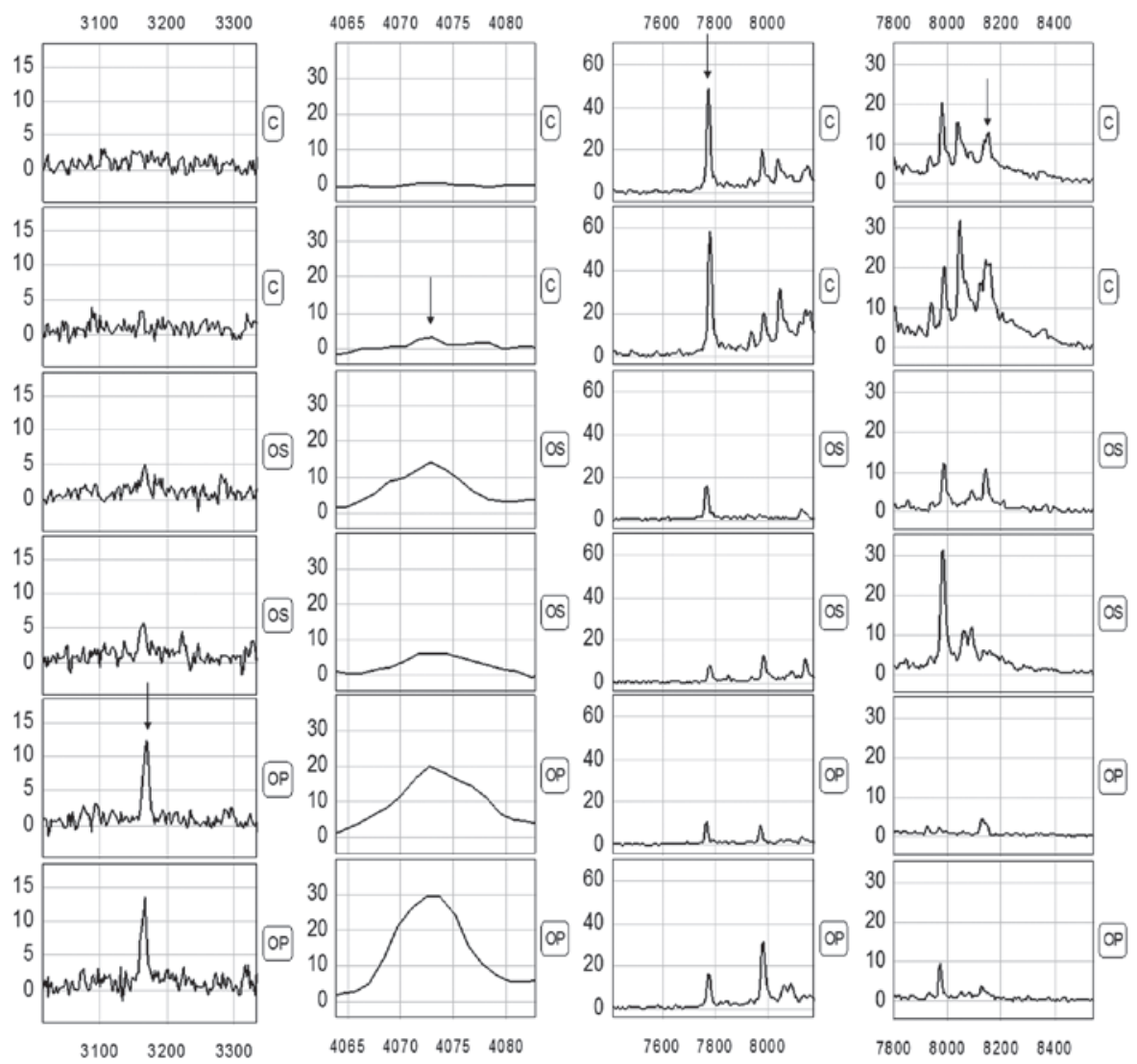

Figure 2. Differential expression of MALDI peaks with $\mathrm{m} / \mathrm{z}$ ratios of 3167.4, 4071.1, 7771.7 and 8140.5 in serum samples from patients with osteoporosis (OS), serious osteoporosis (OP) and the control group (C) is shown.

\section{Results}

Quality control and reproducibility. The quality control (QC) serum sample including 8 mixed serum samples from healthy control subjects with blood type $\mathrm{O}$ ( 4 women and 4 men) was used to determine reproducibility and as a control protein profile for each WCX magnetic beads experiment. The coefficient of variation $(\mathrm{CV})$ for intensity and $\mathrm{m} / \mathrm{z}$ were calculated, based on duplicate sample testing. The intrachip and interchip $\mathrm{CV}$ for intensity were $<10 \%$. The intrachip and interchip CV for $\mathrm{m} / \mathrm{z}$ were $<0.05 \%$. These values showed good reproducibility of spectra over time.

Detection of the protein peaks. Proteomic data from the samples of the training set (comprising 45 PMOP and 40 healthy subjects) were analyzed using the Biomarker Wizard software, version 3.1. Up to 138 protein peaks/spot were detected between $\mathrm{m} / \mathrm{z} 1,000$ and 20,000, while the protein peaks showed the effectiveness of the MALDI technology separation of low-molecular weight proteins $(<20,000)$ (Fig. 1).

Identification of biomarker pattern and construction of diagnostic model. The comparison between various samples showed that the serum profiles from PMOP patients and the control individuals were highly similar in spite of a few intersample variations. Therefore, the number of variations that consistently differentiated these two groups could be considered as potential disease biomarkers. In the present study, we used the Biomarker Wizard function of the proteinchip software to identify clusters of peaks differentially presented in PMOP serum samples compared to healthy controls. We obtained 138 discriminating protein peaks in patient sera. To develop biomarker patterns for the diagnosis of PMOP, 
Table II. Mean signal intensities of various proteins and peptides comparing OS with the healthy control group.

\begin{tabular}{lccc}
\hline $\mathrm{m} / \mathrm{z}$ & PMOP group & Control group & P-value \\
\hline 3167.4 & $4.50 \pm 5.16$ & $0.37 \pm 0.51$ & $6.15 \times 10^{-7}$ \\
7771.7 & $6.08 \pm 8.03$ & $30.77 \pm 24.21$ & $2.01 \times 10^{-6}$ \\
8140.5 & $0.44 \pm 0.52$ & $3.55 \pm 3.83$ & $2.55 \times 10^{-6}$ \\
4071.1 & $5.66 \pm 7.12$ & $0.75 \pm 0.913$ & $3.47 \times 10^{-5}$ \\
\hline
\end{tabular}

OS, osteoporosis; m/z, mass-to-charge ratio; PMOP, postmenopausal osteoporosis.

Table III. Prediction results of the diagnostic model for the OS group.

\begin{tabular}{lcccc}
\hline Groups & $\begin{array}{c}\text { Sample } \\
\text { (group) }\end{array}$ & $\begin{array}{c}\text { No. of } \\
\text { cases }\end{array}$ & $\begin{array}{c}\text { Correctly } \\
\text { classified } \\
\text { cases }\end{array}$ & $\begin{array}{c}\text { Accuracy } \\
(\%)\end{array}$ \\
\hline Training set & PMOP & 45 & 41 & 91.11 \\
& Control & 70 & 65 & 92.86 \\
Blind set & PMOP & 25 & 21 & 84.0 \\
& Control & 40 & 36 & 90.0 \\
\hline
\end{tabular}

OS, osteoporosis; PMOP, postmenopausal osteoporosis.

Table IV. Node variable importance scores of three protein biomarkers.

\begin{tabular}{lcccc}
\hline Node & $\mathrm{m} / \mathrm{z}$ & $\begin{array}{c}\text { Sensitivity } \\
(\%)\end{array}$ & $\begin{array}{c}\text { Specificity } \\
(\%)\end{array}$ & $\begin{array}{c}\text { Variable } \\
\text { importance scores }\end{array}$ \\
\hline 1 & 3167.4 & 66.7 & 69.3 & 100 \\
2 & 4071.1 & 55.6 & 62.7 & 85 \\
3 & 7771.7 & 48.9 & 50.7 & 68 \\
4 & 8140.5 & 44.4 & 42.7 & 55 \\
\hline
\end{tabular}

$\mathrm{m} / \mathrm{z}$, mass-to-charge ratio.

the intensities of the protein peaks in the training set were submitted to BPS. A total of four peaks (3167.4, 4071.1, 7771.7 and 8140.5) with the highest discriminatory power were automatically selected to construct a classification model (Fig. 2 and Table II). The diagnosis model using the combination of the four peaks identified 45 PMOP and 70 controls with a calculated sensitivity of $91.11 \%$ (41/45) and a specificity of $92.86 \%$ (65/70). In the blind test group, 36/40 true control cases were correctly classified, and 21/25 PMOP samples were correctly classified as malignant. These results yield a sensitivity of $84 \%$ and a specificity of $90 \%$ (Table III).

Evaluation of the diagnostic model for PMOP in a blind test. We used 25 samples with PMOP (10 OS and 15 OP) and
40 healthy subjects (10 osteopenia and 30 healthy subjects) to evaluate the PMOP diagnostic model in the blind test. The classification tree discriminated the PMOP from the control samples with a calculated sensitivity of $84 \%$ (21/25) and a specificity of $90 \%$ (36/40) (Table III). The varied importance scores of the three masses as well as the corresponding diagnosis sensitivity and specificity of each mass were summarized in Table IV. m/z 3167.4 had the highest importance score.

\section{Discussion}

PMOP is a common disease that seriously damages the quality of life of older women. This disease usually occurs in women after 5-10 years of menopause, with an estimated incidence of $30-50 \%$ in women aged $>60$ years that increases with age. Osteoporosis-induced fracture has become the major cause of life-shortening disability and teratogenesis in older persons $(5,6)$.

Osteoporosis is a systemic bone disease characterized by reduction in bone mass and tissue microstructure damage, thereby causing reduced bone strength, increased bone fragility and fractures. In adult bones, bone remodeling only emerges in sites with bone resorption, including activation, resorption, formation and quiescence phases. Osteoblasts are responsible for bone formation and osteoclasts for its resorption, and the balance of bone metabolism is maintained by the coordination of those two. Osteoporosis may develop through the following pathways: i) Estrogen promotes calcitonin secretion and inhibits the activity of osteoclasts. Due to the reduction in estrogen secretion in postmenopausal women, calcitonin exhibits a weakening inhibition on osteoclasts, while the increased activity of osteoclasts leads to an increased bone resorption. If bone resorption is greater than bone formation, osteoporosis develops. ii) Lack of estrogen inhibits the secretion of parathyroid hormone. Parathyroid hormone is a catalytic agent that transforms the inactive vitamin $\mathrm{D}$ to active form. Lack of parathyroid hormone secretion leads to a reduction in the production of active vitamin $\mathrm{D}$, causing reduced calcium absorption in intestines; thus, a shortage of raw materials for bone formation leads to osteoporosis.

Currently, the diagnosis of osteoporosis relies on bone density measured by dual-energy X-ray absorptiometry established by WHO in 1994 (7). Such a diagnostic method only reflects the bone mass/unit, and therefore has a number of shortages. The major technical indices are only used for the comparison between healthy adults with the same gender and ethnicity. Due to the limitations of equipments and determination methods, it is impossible to perform large-scale screening of osteoporosis. Currently, the commonly used serum bone markers, such as total procollagen type 1 amino-terminal propeptide, the carboxy-terminal cross-linking telopeptide of type I collagen ( $\beta$-CrossLaps), N-terminal osteocalcin and 25-hydroxyvitamin D3 reflects the changes of bone metabolism $(8,9)$. However, these indicators have a low sensitivity and specificity in the early diagnosis of osteoporosis that does not meet requirements of non-invasive and specific early diagnosis of osteoporosis in the clinical practice. While patients with symptoms visit a doctor, the majority of patients presenting have developed late-stage osteoporosis, in whom relief of pain together with prevention of additional reduc- 
tions in bone mineral density is conducted. However, such treatment has no significant therapeutic effect on severe osteoporosis. Therefore, prevention, early diagnosis, pathogenesis and treatment of osteoporosis have been considered to be high priorities (10).

The present study screened four protein markers with $\mathrm{m} / \mathrm{z}$ of 3167.4, 4071.1, 7771.7 and 8140.5, in which the peaks of the proteins with $\mathrm{m} / \mathrm{z}$ of 3167.4 and 4071.1 were markedly greater in the osteoporosis group compared to the control group, while a significantly higher expression of the proteins with $\mathrm{m} / \mathrm{z}$ of 7771.7 and 8140.5 was detected in the control group. The sensitivity and specificity were 91.11 (41/45) and $92.86 \%(65 / 70)$ in the detection of PMOP using the diagnostic model of the combination of these four markers, suggesting that the four protein markers may be used as indicators for the serological diagnosis of osteoporosis. The established diagnostic model for osteoporosis was validated using the protein fingerprint profiles from 25 osteoporosis cases and 40 controls, and the results showed that the sensitivity and specificity were $84(21 / 25)$ and $90 \%$ (36/40), respectively, for the detection of PMOP.

In conclusion, we have shown that using proteomics approaches, such as magnetic beads and MALDI-TOF-MS in combination with bioinformatics tools, facilitates the establishment of new biomarkers and provide a rapid and mass-accurate mode of analysis for the detection of multiple disease-related proteins in a simultaneous, reproducible and high-throughput manner $(11,12)$. Using the panel of four selected biomarkers, high sensitivity and specificity for the detection of PMOP was achieved. However, in this study, each $\mathrm{m} / \mathrm{z}$ value may represent a number of peptides of similar molecular weight, thus the proteins in the body fluid could not be detected. Therefore, to delineate the structures and functions of the proteins, additional studies are required.

\section{References}

1. Mazzuoli G, Acca M, Pisani D, et al: Annual skeletal balance and metabolic bone marker changes in healthy early postmenopausal women. Bone 26: 381-386, 2000.

2. Wark JD: Osteoporotic fractures: background and prevention strategies. Maturitas 23: 193-207, 1996.

3. Li YZ, Hu CJ, Leng XM, et al: Promising diagnostic biomarkers for primary biliary cirrhosis identified with magnetic beads and MALDI-TOF-MS. Anat Rec (Hoboken) 292: 455-460, 2009.

4. Liu C, Shen J, Pan C, et al: MALDI-TOF MS combined with magnetic beads for detecting serum protein biomarkers and establishment of boosting decision tree model for diagnosis of hepatocellular carcinoma. Am J Clin Pathol 134: 235-241, 2000.

5. European Foundation for Osteoporosis and Bone Disease, National Osteoporosis Foundation: Who are candidates for prevention and treatment for osteoporosis? Osteoporos Int 7: 1-6, 1997.

6. Melton LJ III, Chrischilles EA, Cooper C, et al: Perspective. How many women have osteoporosis? J Bone Miner Res 7: 1005-1010, 1992.

7. Nelson HD, Helfand M, Woolf SH and Allan JD: Screening for postmenopausal osteoporosis: A review of the evidence for the U.S. Preventive Services task force. Ann Intern Med 137: 529-541, 2002.

8. Lappe J, Kunz I, Bendik I, et al: Effect of a combination of genistein, polyunsaturated fatty acids and vitamins D3 and $\mathrm{K} 1$ on bone mineral density in postmenopausal women: a randomized, placebo-controlled, double-blind pilot study. Eur J Nutr: Feb 3, 2012 (Epub ahead of print). doi: 10.1007/s00394012-0304-x.

9. Papierska L, Rabijewski M, Kasperlik-Załuska A and Zgliczyński W: Effect of DHEA supplementation on serum IGF-1, osteocalcin, and bone mineral density in postmenopausal, glucocorticoid-treated women. Adv Med Sci 57: 51-57, 2012.

10. Voelker R: Osteoporosis screening may be needed less often than previously believed. JAMA 307: 654, 2012.

11. Wang QT, Li YZ, Liang YF, et al: Construction of a multiple myeloma diagnostic model by magnetic bead-based MALDI-TOF mass spectrometry of serum and pattern recognition software. Anat Rec (Hoboken) 292: 604-610, 2009.

12. Liu CB, Liang Y, Pan CQ, et al: Proteome study of differential protein expression in HBV-related primary hepatic carcinoma. Chem J Chin Univ 30: 1763-1766, 2009. 